\title{
Nonlocal Coulomb drag in Weyl semimetals
}

\author{
Yuval Baum ${ }^{1,2}$ and Ady Stern ${ }^{1}$ \\ ${ }^{1}$ Department of Condensed Matter Physics, Weizmann Institute of Science, Rehovot 76100, Israel \\ ${ }^{2}$ Institute of Quantum Information and Matter, Department of Physics, California Institute of Technology, Pasadena, California 91125, USA
}

(Received 29 November 2016; published 23 February 2017)

\begin{abstract}
Nonlocality is one of the most striking signatures of the topological nature of Weyl semimetals. We propose to probe the nonlocality in these materials via a measurement of a magnetic-field-dependent Coulomb drag between two sheets of graphene which are separated by a three-dimensional slab of Weyl semimetal. We predict a mechanism of Coulomb drag, based on cyclotron orbits that are split between opposite surfaces of the semimetal. In the absence of impurity scattering between different Weyl nodes, this mechanism does not decay with the thickness of the semimetal.
\end{abstract}

DOI: 10.1103/PhysRevB.95.075141

\section{INTRODUCTION}

Weyl semimetals (WSMs) are a new class of conducting materials, characterized by the nontrivial topological structure of their band structures. These three-dimensional topological semimetals have been the subject of intense recent theoretical and experimental efforts [1-12].

WSMs host an even number of points in the Brillouin zone, known as the Weyl nodes, at which the bulk energy gap closes. The electrons around these nodes disperse relativistically and may be described at low energies by the Weyl Hamiltonian [1-3]. Each Weyl node acts as either a source or a sink of Berry flux. Hence, a single node is stable and cannot be removed. Additionally, any closed two-dimensional (2D) manifold in the three-dimensional Brillouin zone (BZ) which separates regions with different Berry charge must have a nonzero Chern number as it is threaded by a nonzero Berry flux. Thus, as long as Weyl nodes of opposite Berry charge are separated in momentum space, these systems host 2D surface states which form Fermi arcs in the two-dimensional BZ of the surface [2].

Certain iridium pyrochlores and noncentrosymmetric transition metals, e.g., TaAs, have been predicted to host such a topological semimetal phase [5-8]. Following these suggestions, several topological semimetals have been observed experimentally [8-19].

It has been proposed that, when subject to a magnetic field, such materials exhibit unusual physical phenomena, such as the Adler-Bell-Jackiw chiral anomaly [20-22], a unique type of quantum oscillations associated with Fermi-arc surface states [23,24], and various nonlocal transport effects $[25,26]$. In particular, Ref. [26] proposed two experiments that directly probe the exotic topology of these materials: appearance of a magnetic-field-dependent nonlocal dc voltage and sharp resonances in the transmission of electromagnetic waves. Both these effects do not rely on quantum-mechanical phase coherence, which renders them less restrictive in terms of temperature and samples mobility. Nevertheless, they do require challenging experimental setups such as placing contacts in close proximity in the dc proposal and working with a terahertz-frequency radiation in the ac proposal.

In this paper, we propose an alternative dc experiment that does not require any deposition of contacts over the Weyl semimetal: a magnetic-field-dependent nonlocal Coulomb drag measurement. The underlying mechanism in this work is similar to one presented in Refs. [23,25,26]. When a magnetic field is applied perpendicular to surfaces that carry Fermi arcs, electrons traverse cyclotron orbits that connect opposite surfaces of the sample. These unusual intersurface orbits are a source of nonlocal conductivity in these materials; i.e., the application of an electric field along a surface of these materials generates a current along the opposite surface and vice versa. In the following, we provide a prescription which is aimed to detect the emergence of nonlocality via a Coulomb drag experiment.

Standard Coulomb drag setups involve two closely spaced, yet electrically isolated, conductors. Due to the long-range Coulomb interaction between charge carriers in the two conductors, a current in one of the conductors (the active layer) induces a voltage (or a current) in the other conductor (the passive layer). The ratio between the induced voltage in the passive layer and incoming current in the active layer is the drag resistivity [27,28].

Drag measurements may be used to study fundamental properties related to the $e-e$ interaction of diverse condensedmatter systems. Until recently, semiconductor heterostructures, e.g., GaAlAs, with two quantum wells separated by a thin tunnel barrier were the main experimental systems allowing reliable measurements of Coulomb drag [29-31]. The dependence of the drag effect on the layer densities, temperature, and separation distance between the quantum wells has been heavily investigated. In these experiments, the typical separation between the layers was in the range 15-50 $\mathrm{nm}$. In recent years, the appearance of graphene-boron-nitride heterostructures made it possible to investigate drag effects in double-layer graphene down to a separation of $\sim 1 \mathrm{~nm}$ [32-36].

In this work, we consider a scenario where two layers of graphene are separated by a three-dimensional (3D) slab of WSM. The graphene layers are coupled to the WSM only via the Coulomb interaction; i.e., the top and bottom graphene layers generate drag force on the top and bottom surfaces of the WSM, respectively. In the absence of an external magnetic field the system may be thought of as two decoupled standard Coulomb drag systems; each one contains a layer of graphene which is Coulomb coupled to a surface of a WSM. Since the WSM is metallic, the coupling between these two systems is nonzero; nonetheless, for macroscopic slabs this coupling is extremely small as we show below. On the contrary, in the presence of an external magnetic field, the nonlocality in the 


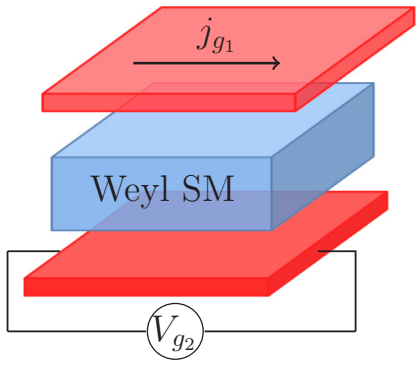

(a)

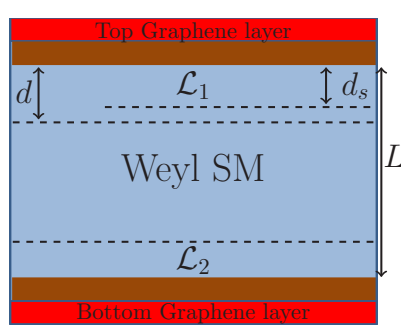

(b)
FIG. 1. (a) Drag setup: two layers of graphene (red) are placed above and below a 3D Weyl semimetal. The graphene layers and the Weyl semimetal are separated by insulating layers (brown). The graphene layers are coupled to the Weyl metal only via the local Coulomb interaction. Current is pushed on the top layer while the voltage on the bottom layer is measured. (b) A sketch of the relevant length scales in the problem. $L$ is the thickness of the WSM, $d_{s}$ is the extent of the surface states, and $d$ is the thickness of the layer at which the drag coupling is nonzero. We denote the layers at which the surface states exist by $\mathcal{L}_{1}$ and $\mathcal{L}_{2}$.

WSM yields a strong coupling between the two drag systems and current in the top graphene layer leads to a non-negligible voltage buildup on the distant lower graphene layer.

The source of the nonlocal drag is rather simple. As long as current flows in the top graphene layer, a portion of its momentum is transferred to the top surface of the WSM, due to the existence of a frictional Coulomb force. For a standard two-dimensional electron gas (2DEG) in an open circuit configuration, this momentum transfer is translated into a voltage buildup. However, as shown in Refs. [23,26], for relatively clean samples and in the presence of an external magnetic field the current on the top surface follows the intersurface cyclotron orbits leading to a circulating current throughout the 3D sample. In particular, a current on the bottom surface is inevitable for clean samples. When the nonlocal conductivity is much larger than the local one, the current on the bottom surface flows with a similar magnitude and an opposite direction to the current on the top surface. As before, due to the existence of a frictional Coulomb force between the bottom surface of the WSM and the bottom graphene layer, a momentum transfer to the second graphene layer occurs. This momentum transfer is translated into a voltage buildup on the passive graphene layer. The ratio between this voltage and the current on the top layer is the nonlocal drag coefficient.

\section{SETUP AND TRANSPORT EQUATIONS}

In this section, we describe the experimental setup and the procedure of finding the nonlocal drag coefficient. The setup we consider is depicted in Fig. 1(a). Two layers of graphene (red) are placed above and below a 3D Weyl metal. The graphene layers are coupled to the WSM only via the Coulomb interaction; i.e., the tunneling between the graphene layers and the WSM is assumed to be zero. A current $j_{g_{1}}$ is pushed in the first graphene layer while the voltage (electric field) buildup on the second graphene layer, $V_{g_{2}}\left(\epsilon_{g_{2}}\right)$, is measured. The ratio between these two quantities, $R_{D}=\epsilon_{g_{2}} / j_{g_{1}}$, is the nonlocal drag coefficient.

We analyze the semiclassical transport equations, assuming that the relevant transport parameters are known. For simplicity, we assume that the two graphene layers are identical. We denote their 2D density by $n_{g}$ and their 2D intralayer resistivity by $\rho_{g}$. Moreover, at this stage, we assume that the Coulomb coupling between the WSM and the two graphene layers is symmetric and can be characterized by a single $2 \mathrm{D}$ drag resistivity $\rho_{d}$. The form of $\rho_{d}$ is given in Appendix C. Finally, we denote the 3D density and the $3 \mathrm{D}$ resistivity of the WSM by $n_{3 \mathrm{D}}$ and $\rho_{3 \mathrm{D}}$, respectively.

A standard double-layer Coulomb drag system is well described by two coupled Drude-like equations of motion,

$$
\begin{aligned}
& m_{1} \frac{d \mathbf{v}_{1}}{d t}=e\left(\mathbf{E}_{1}+\mathbf{v}_{1} \times \mathbf{B}\right)-\frac{m_{1} \mathbf{v}_{1}}{\tau_{1}}-\hbar \gamma n_{2}\left(\mathbf{v}_{1}-\mathbf{v}_{2}\right), \\
& m_{2} \frac{d \mathbf{v}_{2}}{d t}=e\left(\mathbf{E}_{2}+\mathbf{v}_{2} \times \mathbf{B}\right)-\frac{m_{2} \mathbf{v}_{2}}{\tau_{2}}-\hbar \gamma n_{1}\left(\mathbf{v}_{2}-\mathbf{v}_{1}\right),
\end{aligned}
$$

where $\mathbf{B}$ is the magnetic field, $e$ is the electric charge, and $\mathbf{v}_{i}$, $m_{i}, n_{i}$, and $\mathbf{E}_{i}$ are the drift velocity, effective mass, density, and electric field in the $i$ 's layer, respectively. $\tau_{i}$ is the $i$ 's layer intralayer momentum relaxation time due to impurity scattering while the dimensionless parameter $\gamma$ is related to the mutual interlayer momentum relaxation rate due to the Coulomb friction. The drag resistivity arising from Eq. (1) is $\hbar \gamma / e^{2}$. In the following, we assume steady state; i.e., the left-hand side above is zero.

In the setup we propose, each graphene layer is Coulomb coupled locally to the adjacent surface of the 3D WSM, and the WSM, similar to Ref. [26], has both a local and a nonlocal conductivity. We denote the three-dimensional electric field and the current density in the WSM by $\mathbf{E}(x, y, z)$ and $\mathbf{J}(x, y, z)$. The current density and electric field in the WSM must satisfy Kirchhoff's rules and Ohm's law,

$$
\begin{aligned}
\nabla \cdot \mathbf{J} & =\nabla \times \mathbf{E}=0, \\
\mathbf{E} & =\hat{\rho} \mathbf{J},
\end{aligned}
$$

where the resistivity matrix includes both the local and the nonlocal parts. In the limit where the thickness of the WSM (extent in the $z$ direction) is much smaller than its extent in the other directions, we may assume that the bulk values of $\mathbf{E}$ and $\mathbf{J}$ do not depend on $x$ and $y$. Hence, the requirement $\nabla \cdot \mathbf{J}=0$ implies that $\partial_{z} J_{z}=0$ and therefore $J_{z}=0$, since it must be zero on the boundaries. Moreover, since the WSM is in an open circuit configuration, the net current in the $3 \mathrm{D}$ bulk must be zero, i.e.,

$$
\int d z J_{x}(z)=\int d z J_{y}(z)=0,
$$

where the integration is over the thickness of the WSM slab. Additionally, the requirement $\nabla \times \mathbf{E}=0$ implies that $\mathbf{E}=$ const.

Next, we denote the two-dimensional electric fields and the current densities in the $i$ 's graphene layer by $\mathbf{j}_{g_{i}}(x, y)$ and $\boldsymbol{\epsilon}_{g_{i}}(x, y)$. The currents in the graphene layers are taken to be controlled quantities in the experiment while the spatially independent electric fields are the unknowns which should be determined. 
The above analysis points out that we are left with five unknown quantities, three uniform fields $\boldsymbol{\epsilon}_{g_{1}}, \boldsymbol{\epsilon}_{g_{2}}$, and $\mathbf{E}$ and two $z$-dependent currents, $\mathbf{J}_{x}(z)$ and $\mathbf{J}_{y}(z)$. In order to determine these unknown quantities, we invoke Ohm's law for the combined graphene-WSM system. In Appendix B we show that the effect of the intralayer Hall effect on the nonlocal drag is negligible. Hence, we ignore the Lorentz force in Eq. (1).

The electric field in the graphene layers is determined only by the intralayer scattering and the local Coulomb drag:

$$
\begin{array}{r}
\boldsymbol{\epsilon}_{g_{1}}=\left(\rho_{g}+\frac{n_{3 \mathrm{D}}}{n_{g}} \int d z^{\prime} \rho_{d 1}\left(z^{\prime}\right)\right) \mathbf{j}_{g_{1}}-\int d z^{\prime} \rho_{d 1}\left(z^{\prime}\right) \mathbf{J}\left(z^{\prime}\right) \\
\boldsymbol{\epsilon}_{g_{2}}=\left(\rho_{g}+\frac{n_{3 \mathrm{D}}}{n_{g}} \int d z^{\prime} \rho_{d 2}\left(z^{\prime}\right)\right) \mathbf{j}_{g_{2}}-\int d z^{\prime} \rho_{d 2}\left(z^{\prime}\right) \mathbf{J}\left(z^{\prime}\right)
\end{array}
$$

where $\rho_{d i}(z)$ is the local drag resistivity that couples the $i$ 's graphene layer to a fixed $z$ layer of the WSM, where the integrals run over the thickness of the sample. Both $\rho_{d 1}$ and $\rho_{d 2}$ are local quantities and, hence, vanish away from the top and bottom surfaces, respectively. Since $\mathbf{j}_{g_{1}} /\left(e n_{g}\right)$ is the velocity in the graphene layer and $\mathbf{J}(z) /\left(e n_{3 \mathrm{D}}\right)$ is the velocity in the WSM, it is evident, as expected, that when the velocities are equal the drag is zero.

In the WSM, there are three contributions to the resistivity: the impurity scattering, the Coulomb drag with both graphene layers, and an additional contribution coming from the intersurface cyclotron orbits as derived in Ref. [26]. Overall, Ohm's law in the WSM is given by

$$
\begin{aligned}
\mathbf{E}= & \left(\rho_{3 \mathrm{D}}+\frac{n_{g}}{n_{3 \mathrm{D}}}\left[\rho_{d 1}(z)+\rho_{d 2}(z)\right]\right) \mathbf{J}(z) \\
& -\rho_{d 1}(z) \mathbf{j}_{g 1}-\rho_{d 2}(z) \mathbf{j}_{g 2}+\int d z^{\prime} \rho_{w}\left(z, z^{\prime}\right) \mathbf{J}\left(z^{\prime}\right),
\end{aligned}
$$

where $\rho_{w}\left(z, z^{\prime}\right)$ is the contribution of the intersurface cyclotron orbits to the resistivity of the WSM. In general, $\rho_{w}\left(z, z^{\prime}\right)$ contains both local and nonlocal contributions.

For concreteness, we denote the thickness of the WSM by $L$. The Coulomb interaction between the graphene layers and the WSM bulk vanishes exponentially away from the surface, where the decaying scale is determined by the ThomasFermi screening length. Therefore, the coupling between the graphene layers and the WSM is highly local. For simplicity, we assume that the coupling is uniform inside thin layers near the surfaces of the WSM and zero outside of these layers; i.e.,

$$
\begin{aligned}
& \rho_{d 1}= \begin{cases}\rho_{d}, & 0<z<d \\
0, & \text { otherwise },\end{cases} \\
& \rho_{d 2}= \begin{cases}\rho_{d}, & L-d<z<L \\
0, & \text { otherwise },\end{cases}
\end{aligned}
$$

where we denoted the thickness of the layers in which the drag is effective by $d$. We assume $d \ll L$. In the presence of an external magnetic field, nonlocal effects connect the top and bottom surfaces of the WSM. We denote the extent of the surface states by $d_{s} \ll L$. All these scales are depicted in
Fig. 1(b). With these assumptions, we may write $\rho_{w}\left(z, z^{\prime}\right)$ as

$$
\frac{\rho_{w}\left(z, z^{\prime}\right)}{d_{s} \rho_{3 \mathrm{D}}}= \begin{cases}\Gamma, & z, z^{\prime} \in \mathcal{L}_{1} \\ \Gamma, & z, z^{\prime} \in \mathcal{L}_{2} \\ -\Gamma, & z \in \mathcal{L}_{1}, z^{\prime} \in \mathcal{L}_{2} \\ -\Gamma, & z \in \mathcal{L}_{2}, z^{\prime} \in \mathcal{L}_{1},\end{cases}
$$

where $\mathcal{L}_{1}$ is the layer defined by $z \in\left[0, d_{s}\right], \mathcal{L}_{2}$ is the layer defined by $z \in\left[L-d_{s}, L\right]$, and the dimensionless parameter $\Gamma$ is specified below.

In order to solve Eqs. (4) and (5) with the appropriate boundary conditions and the constraint of Eq. (3), we discretize the 3D WSM to $N$ quasi-2D layers. The full description of the solution appears in Appendix A.

Before diving into the details of the exact solution, it is instructive to qualitatively examine the case without nonlocality which is applicable when no magnetic field is present. In this case, a current in the top graphene layer leads to a current, in the same direction, in the top layer of thickness $d$ of the WSM. For weak drag this current is given by $j_{\text {top }}=\left(\rho_{d} j_{g_{1}}\right) / \rho_{3 \mathrm{D}}$. Away from the top surface, Eq. (5) implies that the current in the bulk is uniform. However, Eq. (3) requires that the total current is zero. Hence, an opposite current must flow in the bulk of the WSM in order to cancel the current in the top drag layer. The magnitude of this current must scale as $d / L$. In turn, this opposite current leads to voltage buildup on the second graphene layer due to its Coulomb coupling. The electric field in the bottom graphene layer is determined by the current in the bottom drag layer of the WSM, $\epsilon_{g_{2}}=\rho_{d} j_{\text {bot }}$. Finally by using the currents ratio $j_{\text {bot }} / j_{\text {top }}=d / L$ we expect $R_{D} \approx\left(\frac{d}{L}\right) \frac{\rho_{d}^{2}}{\rho_{3 \mathrm{D}} / d}$. In the next section we show that the effect of nonlocality is to increase the current flow near the bottom surface, hence increasing the nonlocal drag resistivity.

\section{THE NONLOCAL DRAG COEFFICIENT}

We express the nonlocal drag coefficient by the three length scales above and the following quantities: the local sheet resistivity $r_{0}=\rho_{3 \mathrm{D}} / L$, the dimensionless nonlocal parameter $\Gamma$, and the effective density ratio $\xi=\frac{n_{3 D} L}{n_{g}}$, which generically is much larger than unity. Solving for the nonlocal drag coefficient $R_{D}=\epsilon_{g_{2}} / j_{g_{1}}$ and employing the above notations yields the following lengthy expression whose limits we aim to examine:

$$
R_{D}=\frac{d_{s} \xi^{2} r_{0} \rho_{d}^{2}\left(\frac{d}{d_{s}}\left(\rho_{d}+\xi r_{0}\right)+\Gamma\left[A \rho_{d}+B \xi r_{0}\right]\right)}{L\left(\rho_{d}+\xi r_{0}\right)\left[A \rho_{d}+\frac{L}{d} \xi r_{0}\right]\left[\rho_{d}+\xi r_{0}(1-2 \Gamma)\right]},
$$

where $A=\frac{L}{d}-2$ and $B=\frac{L}{d}-\frac{2 d}{d_{s}}$.

In the following parts, we investigate the nonlocal drag coefficient in the relevant limits. For macroscopic samples we may assume $\frac{L}{d} \gg 1$ and $\rho_{d} \ll \xi r_{0}$. In this limit Eq. (8) becomes:

$$
R_{D} \approx \frac{d d_{s} \xi \rho_{d}^{2}}{L^{2}} \frac{\frac{d}{d_{s}}+\Gamma \frac{L}{d}}{\rho_{d}+\xi r_{0}(1-2 \Gamma)} .
$$

In the absence of an external magnetic field and in the limit of weak drag, Eq. (8) reduces to the expected result, as discussed in the previous section.

We now turn to the more interesting case in which $\Gamma \neq$ 0 , i.e., in the presence of an external magnetic field. It is 
constructive to introduce the notation

$$
r_{0}(1-2 \Gamma)=\frac{r_{0} r_{w}}{2 r_{0}+r_{w}},
$$

with $L r_{w}=1 / \sigma_{w}$, where $\sigma_{w}$ is the nonlocal conductivity as derived in Ref. [26]. In the local case $r_{w}$ diverge to infinity and therefore $\Gamma=0$, whereas when the nonlocal conductivity is much larger than the local one then $r_{w} \ll r_{0}$ and therefore $\Gamma \rightarrow 1 / 2$. Employing the above notation, the nonlocal drag coefficient is given by

$$
R_{D} \approx \frac{\xi^{2} r_{0} \rho_{d}^{2}\left[\left(\frac{d}{L}\right)^{2}+\Gamma\left(\frac{d}{L}\right)\right]}{\left(\rho_{d}+\xi r_{0}\right)\left[\rho_{d}+\xi \frac{r_{0} r_{w}}{2 r_{0}+r_{w}}\right]} \approx \frac{\Gamma n_{3 \mathrm{D}} d_{s}}{n_{g}} \frac{\rho_{d}^{2}}{\rho_{d}+\xi \frac{r_{0} r_{w}}{2 r_{0}+r_{w}}}
$$

For low magnetic fields, the nonlocal conductivity is small compared to the local one, i.e., $r_{w} \gg r_{0}$. In this limit and assuming as before $\rho_{d} \ll \xi r_{0}$, Eq. (11) yields

$$
R_{D} \approx \Gamma \frac{d_{s}}{L} \frac{\rho_{d}^{2}}{r_{0}}=\Gamma \frac{\rho_{d}^{2}}{\rho_{3 \mathrm{D}} / d_{s}}+O\left(\frac{d}{L}\right) .
$$

Remarkably, we find that in the presence of an external magnetic field the leading term in $R_{D}$ becomes independent of the WSM thickness $L$, consensuses of the intersurface cyclotron orbits. This result is valid as long as the circulating intersurface current exists. As shown in Refs. [23,26], this is the case whenever the thickness of the WSM is smaller than the internode-transport scattering length. In these materials it is expected that the internode scattering length should be much smaller than the single-particle scattering length [25].

If the magnetic field is further increased, the nonlocal conductivity becomes larger than the local one; i.e., $r_{w} \ll r_{0}$ and $\Gamma \rightarrow \frac{1}{2}$. In this limit Eq. (11) becomes

$$
R_{D} \approx \frac{n_{3 \mathrm{D}} d_{s}}{n_{g}} \frac{\rho_{d}^{2}}{2 \rho_{d}+\xi r_{w}}+O\left(\frac{d}{L}\right),
$$

which may be split into two cases: for $\rho_{d} \ll \xi r_{w}$,

$$
R_{D} \approx \frac{d_{s}}{L} \frac{\rho_{d}^{2}}{r_{w}}+O\left(\frac{d}{L}\right)
$$

which is similar to Eq. (12) only with $\Gamma / r_{0}$ being replaced by $1 / r_{w}$. Remember that in this limit $r_{w} \ll r_{0} / \Gamma$.

On the other hand, if $r_{w}$ is further reduced such that $\xi r_{w} \ll$ $\rho_{d}$, then

$$
R_{D} \approx \frac{n_{3 \mathrm{D}} d_{s}}{n_{g}} \frac{\rho_{d}}{2}+O\left(\frac{d}{L}\right)
$$

Surprisingly, in this case $R_{D} \propto \rho_{d}$. It is instructive to reproduce Eq. (15) in the nonsymmetric case where the drag resistivity on the top and the drag resistivity on the bottom are different. We denote the different local drag resistivities by $\rho_{d 1}$ and $\rho_{d 2}$, respectively. In the nonsymmetric case Eq. (15) becomes

$$
R_{D} \approx \frac{n_{3 \mathrm{D}} d_{s}}{n_{g}} \frac{\rho_{d_{1}} \rho_{d_{2}}}{\rho_{d_{1}}+\rho_{d_{2}}}=\frac{n_{3 \mathrm{D}} d_{s}}{n_{g}}\left[\frac{1}{\rho_{d_{1}}}+\frac{1}{\rho_{d_{2}}}\right]^{-1},
$$

which implies that $R_{D}$ is dominated by the minimal local drag resistivity. It worth noting that the nonlocal drag coefficient in Eq. (15) may be larger than the local drag resistivity.
TABLE I. Typical mobilities (of thin films) in units of $\mathrm{V}^{-1} \mathrm{~s}^{-1} \mathrm{~cm}^{2}$.

\begin{tabular}{lrrr}
\hline \hline & CdAs & NbP & \multicolumn{1}{c}{ TaAs } \\
\hline$T<1.5 \mathrm{~K}$ & $9 \times 10^{6}$ & $5 \times 10^{6}$ & $5 \times 10^{5}$ \\
$T \sim 130 \mathrm{~K}$ & $4.2 \times 10^{4}$ & $7 \times 10^{3}$ & $1.5 \times 10^{3}$ \\
$T \sim 300 \mathrm{~K}$ & $1.5 \times 10^{4}$ & $3 \times 10^{2}$ & \\
\hline \hline
\end{tabular}

\section{ESTIMATE OF SCALES}

We may consider both Dirac metals (e.g., CdAs) and Weyl metals (e.g., TaAs, TaP, NbAs, and NbP). In these materials, the typical bulk density is in the range $n_{3 \mathrm{D}}=3 \times 10^{18}$ to $3 \times$ $10^{19} \mathrm{~cm}^{-3}$, while a typical density of graphene sheets is in the range $n_{g}=1 \times 10^{11}$ to $1 \times 10^{12} \mathrm{~cm}^{-2}$. The mobilities of WSM may vary dramatically between the different materials [9,37-39]; see Table I.

As a result, at moderate temperatures $(20<T<150 \mathrm{~K})$ the typical $3 \mathrm{D}$ resistivity of these materials is in the range $\rho_{3 \mathrm{D}}=5-100 \mu \Omega \mathrm{cm}$.

With these parameters in hand we may estimate the relevant transport parameters of our system. We consider a thin sample of a WSM with $L \sim 100 \mathrm{~nm}$, where both $d$ and $d_{s}$ are in the nanometer scale $(\sim 1-5 \mathrm{~nm})$. Moreover, $r_{0}=\frac{\rho_{3 \mathrm{D}}}{L}=0.5-10 \Omega$ and $r_{0} \xi=\left(e n_{g} \mu\right)^{-1}=30-10^{3} \Omega$, where $\mu$ is the mobility of the WSM. At moderate temperatures, typical values for the local drag resistivity in graphene systems (away from the dual neutrality point) are in the range $\rho_{d} \sim 0.1-10 \Omega$ [32]. See Appendix $\mathrm{C}$ for an estimation of $\rho_{d}$.

Employing these parameters, the ratio of $R_{D}$ in the presence of nonlocality $(\Gamma \neq 0)$ and $R_{D}$ in the absence of nonlocality $(\Gamma=0)$ appears in Fig. 2. As evident, the nonlocal drag coefficient increases by orders of magnitude as a function of $\Gamma$. In particular, while the value of $R_{D}$ for $\Gamma=0$ is practically unmeasurable (of the order of $\mu \Omega$ ), it becomes of the order of $\Omega$ for $\Gamma \sim 1 / 2$.

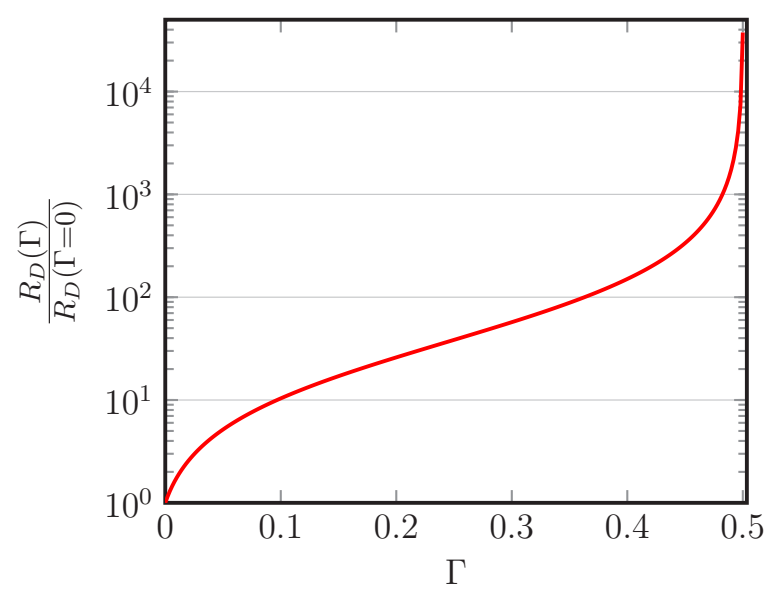

FIG. 2. The ratio of $R_{D}$ in the presence of nonlocality $(\Gamma \neq 0)$ and $R_{D}$ in the absence of nonlocality $(\Gamma=0)$. Here, $L=100 \mathrm{~nm}$, $d_{s}=3 \mathrm{~nm}, d=2 \mathrm{~nm}, r_{0}=5 \Omega, \rho_{d}=0.5 \Omega$, and $\xi=100$. For $\Gamma=0$ the drag coefficient is $R_{D}=2 \times 10^{-5} \Omega$ and for $\Gamma=1 / 2$ it is $R_{D}=$ $0.75 \Omega$. 


\section{CONCLUSIONS}

We have shown that the nonlocal conductivity in WSM which arises from intersurface cyclotron orbits may be detected in a Coulomb drag measurement. We demonstrated that, when the nonlocal conductivity is nonzero, a measurable drag resistance is expected between two layers of graphene which are separated by hundreds of nanometers. Such an unprecedented strong signal of drag in these scales provides clear-cut evidence for the existence of nonlocal effects and, therefore, for the special topological structure of these materials.

While in WSM with $C_{4}$ symmetry, the nonlocal drag is expected to be isotropic, in WSMs that break the $C_{4}$ symmetry, the nonlocal drag is expected to be highly nonisotropic, which is a direct measure of the unique arc structure of the surface states. Furthermore, similar to Ref. [26], the semiclassical origin of this effect renders it more robust and experimentally accessible than quantum effects.

It is also worth mentioning that it may be possible to use the drag setup only along the top surface of the Weyl semimetal, while the detection of the nonlocal current may be done by a direct measurement of the voltage buildup on the bottom surface of the Weyl semimetal.

\section{ACKNOWLEDGMENTS}

We thank J. Eisenstein and Y. Oreg for useful discussions. We also acknowledge support from the European Research Council under the European Union's Seventh Framework Program (Grant No. FP7/2007-2013)/ERC Project MUNATOP, the DFG (CRC/Transregio 183, Grant No. EI 519/7-1), Minerva Foundation, and the U.S.-Israel BSF. Y.B. is grateful for support from the Institute of Quantum Information and Matter.

\section{APPENDIX A: SOLUTION OF THE TRANSPORT EQUATIONS}

In order to solve Eqs. (4) and (5) with the appropriate boundary conditions and the constraint of Eq. (3), we discretize the $3 \mathrm{D}$ WSM to $N$ quasi-2D layers. Each such layer is characterized by its own density $n=n_{3 \mathrm{D}} L / N$ and its own quasi-2D intralayer resistivity $\rho_{0}=\rho_{3 \mathrm{D}} N / L$. We denote by $N_{d}$ and $N_{s}$ the number of layers within a distance $d$ and $d_{s}$ from one of the surfaces, respectively. Each layer within a distance $d$ from the top or bottom surface is coupled the top or bottom graphene layer according to Eq. (1). In the presence of an external magnetic field, each layer within a distance $d_{s}$ from one of the surfaces is coupled to all the other layers within a distance $d_{s}$ from the opposite surface via the quasi-2D nonlocal resistivity, which we denote by $\rho_{w}$.

In Appendix B we show that the effect of the intralayer Hall effect on the nonlocal drag is negligible. Hence, we ignore the Lorentz force in Eq. (1). Without the loss of generality, we assume that $N_{s} \leqslant N_{d}$. Similar to the main text, we introduce the dimensionless density ratio $\xi=\frac{n_{3 \mathrm{D}} L}{n_{g}}$, and the dimensionless nonlocal parameters $\Gamma=\frac{\rho_{0}}{2 \rho_{0}+\rho_{w}}$ and $v=\frac{\rho_{0}}{\rho_{0}+\rho_{w}}$.

The steady-state equations for the longitudinal electric fields and current densities in the two graphene layers and the $N$ layers of the WSM are

$$
\begin{aligned}
& E_{g 1}=\left(\rho_{g}+N_{d} \frac{n}{n_{g}} \rho_{d}\right) j_{g_{1}}-\rho_{d} \sum_{i=1}^{N d} j_{i}, \\
& E_{k_{1}}=\left(\rho_{0} \gamma+\frac{N}{\xi} \rho_{d}\right) j_{k_{1}}-\rho_{d} j_{0}+\frac{\Gamma \rho_{0}}{N_{g}} \sum_{i=N-N_{g}}^{N} j_{i}, \\
& E_{k_{2}}=\left(\rho_{0}+\frac{N}{\xi} \rho_{d}\right) j_{k_{2}}-\rho_{d} j_{0}, \\
& E_{k_{3}}=\rho_{0} j_{k_{3}}, \\
& E_{k_{4}}=\left(\rho_{0} \gamma+\frac{N}{\xi} \rho_{d}\right) j_{k_{4}}-\rho_{d} j_{0}+\frac{\Gamma \rho_{0}}{N_{g}} \sum_{i=1}^{N_{g}} j_{i}, \\
& E_{g 2}=\left(\rho_{g}+N_{d} \frac{n}{n_{g}} \rho_{d}\right) j_{g_{2}}-\rho_{d} \sum_{i=N-N_{d}}^{N} j_{i},
\end{aligned}
$$

where $k_{1} \in\left[1, N_{g}\right], k_{2} \in\left[N_{g}+1, N_{d}\right] \vee\left[N-N_{d}+1, N-\right.$ $\left.N_{g}\right], k_{3} \in\left[1+N_{d}, N-N_{d}\right], k_{4} \in\left[N-N_{g}+1, N\right]$, and $\gamma=$ $1-\Gamma(1+v)$. Equations (A1) may be recast in a matrix form (discrete Ohm's law):

$$
\mathcal{E}=\hat{\rho} \mathcal{J}
$$

where we defined $\mathcal{E}=\left(E_{g_{1}}, E_{1}, \ldots, E_{N}, E_{g_{2}}\right)^{T}, \quad \mathcal{J}=$ $\left(j_{g_{1}}, j_{1}, \ldots, j_{N}, j_{g_{2}}\right)^{T}$ and $\hat{\rho}$ is the $(N+2) \times(N+2)$ resistivity matrix of the system. In order to solve Eq. (A2) we must specify $N+2$ of the above quantities. In the proposed setup the current on the upper graphene layer is fixed, $j_{g_{1}}$. Additionally, if the lower graphene layer and the WSM are in an open circuit configuration, then $j_{g_{2}}=0$ and $\sum_{i=1}^{N} j_{i}=0$. The last condition automatically satisfies $\nabla \cdot \mathbf{j}=0$ inside the Weyl conductor. Finally, we must demand that $\nabla \times \mathbf{E}=0$ inside the WSM. This translates to $E_{1}=E_{2}=\cdots=E_{N}$. Forcing these constraints, Eq. (A2) may be solved uniquely and all the physical and meaningful quantities have a well-defined $N \rightarrow \infty$ limit.

\section{APPENDIX B: INCLUDING THE INTRALAYER HALL CONDUCTIVITY}

Semiclassically, the local intralayer conductivities are given by $\sigma_{x x}=\frac{\sigma_{0}}{1+\phi^{2}}$ and $\sigma_{x y}=\frac{\phi \sigma_{0}}{1+\phi^{2}}$. Within the framework of Drude, $\phi=\frac{\sigma_{x y}}{\sigma_{x x}}=\omega_{c} \tau$; however, here we treat $\phi$ as a tuning parameter that captures the strength of the Hall effect.

It is instructive to construct the full resistivity tensor for two layers in the presence of a nonlocal conductivity and a Hall conductivity. The full conductivity matrix is given by

$$
\hat{\sigma}=\left(\begin{array}{cccc}
\sigma_{x x}+\sigma_{W} & -\sigma_{x y} & -\sigma_{W} & 0 \\
\sigma_{x y} & \sigma_{x x}+\sigma_{W} & 0 & -\sigma_{W} \\
-\sigma_{W} & 0 & \sigma_{x x}+\sigma_{W} & -\sigma_{x y} \\
0 & -\sigma_{W} & \sigma_{x y} & \sigma_{x x}+\sigma_{W}
\end{array}\right)
$$




$$
=\left(\begin{array}{cccc}
\frac{\sigma_{0}}{1+\phi^{2}}+\sigma_{W} & -\frac{\phi \sigma_{0}}{1+\phi^{2}} & -\sigma_{W} & 0 \\
\frac{\phi \sigma_{0}}{1+\phi^{2}} & \frac{\sigma_{0}}{1+\phi^{2}}+\sigma_{W} & 0 & -\sigma_{W} \\
-\sigma_{W} & 0 & \frac{\sigma_{0}}{1+\phi^{2}}+\sigma_{W} & -\frac{\phi \sigma_{0}}{1+\phi^{2}} \\
0 & -\sigma_{W} & \frac{\phi \sigma_{0}}{1+\phi^{2}} & \frac{\sigma_{0}}{1+\phi^{2}}+\sigma_{W}
\end{array}\right) .
$$

Inverting this matrix yields the following resistivities:

$$
\begin{gathered}
\rho_{x x}=\frac{1}{\sigma_{0}} \frac{1-\Gamma\left(1-\phi^{2}\right)}{1+(2 \Gamma \phi)^{2}}, \\
\rho_{x y}=\frac{\phi}{\sigma_{0}} \frac{1-2 \Gamma+2 \Gamma^{2}\left(1+\phi^{2}\right)}{1+(2 \Gamma \phi)^{2}}, \\
\rho_{x x}^{N L}=\frac{\Gamma}{\sigma_{0}} \frac{1-\phi^{2}(1-4 \Gamma)}{1+(2 \Gamma \phi)^{2}}, \\
\rho_{x y}^{N L}=\frac{2 \Gamma \phi}{\sigma_{0}} \frac{1-\Gamma\left(1-\phi^{2}\right)}{1+(2 \Gamma \phi)^{2}} .
\end{gathered}
$$

For $\phi=0$ we get

$$
\begin{aligned}
\rho_{x x} & =\frac{1}{\sigma_{0}}(1-\Gamma), \quad \rho_{x y}=0, \\
\rho_{x x}^{N L} & =\frac{\Gamma}{\sigma_{0}}, \quad \rho_{x y}^{N L}=0,
\end{aligned}
$$

as we got in the sections above. For $\Gamma=0$, we get

$$
\begin{aligned}
\rho_{x x} & =\frac{1}{\sigma_{0}}, \rho_{x y}=\frac{\phi}{\sigma_{0}}, \\
\rho_{x x}^{N L} & =0, \rho_{x y}^{N L}=0,
\end{aligned}
$$

as expected. This resistivity tensor can be generalized to $N$ layers. Including the effect of drag as before, we get a similar relation to Eq. (A2),

$$
\left(\begin{array}{c}
E_{g 1 x} \\
E_{g 1 y} \\
E_{1 x} \\
E_{1 y} \\
\vdots \\
E_{N x} \\
E_{N y} \\
E_{g 2 x} \\
E_{g 2 y}
\end{array}\right)=\hat{\rho}\left(\begin{array}{c}
j_{g 1 x} \\
j_{g 1 y} \\
j_{1 x} \\
j_{1 y} \\
\vdots \\
j_{N x} \\
j_{N y} \\
j_{g 2 x} \\
j_{g 2 y}
\end{array}\right),
$$

where now $\hat{\rho}$ is the $2(N+2) \times 2(N+2)$ resistivity matrix of the system. As before, in order to solve Eq. (B8) we now must specify $2(N+2)$ of the above quantities. The current on the upper graphene layer is fixed, $j_{g 1 x}$. Additionally, if the lower graphene layer and the WSM are in an open circuit configuration, then $j_{g_{2} x}=j_{g_{2} y}=j_{g_{1} y}=0$ and $\sum_{i=1}^{N} j_{i x}=0$ and $\sum_{i=1}^{N} j_{i y}=0$. The last two conditions give $\nabla \cdot \mathbf{j}=0$ in the Weyl conductor. Finally, we must demand that inside the WSM $\nabla \times \mathbf{E}=0$. This translates to $E_{1 x}=E_{2 x}=\cdots=E_{N x}$ and $E_{1 y}=E_{2 y}=\cdots=E_{N y}$. With these constraints, Eq. (B8) may be solved uniquely. The longitudinal nonlocal drag coefficient

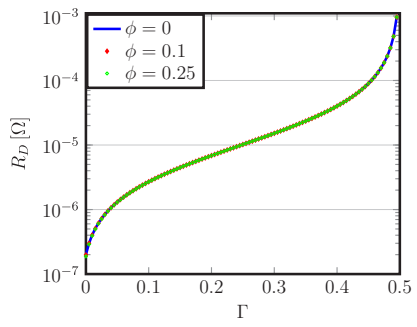

(a)

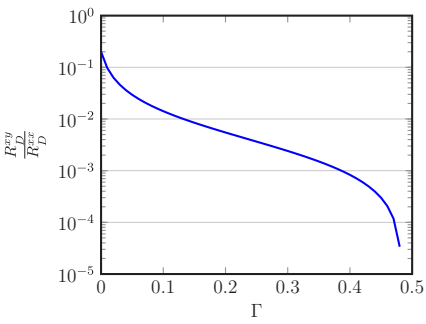

(b)
FIG. 3. (a) $R_{D}^{x x}$ as a function of $\Gamma$ for three different values of $\phi$. (b) The ratio $\Phi$ as a function of $\Gamma$ for $\phi=0.2$. In both (a) and (b), $L=100 \mathrm{~nm}, d_{s}=d=1 \mathrm{~nm}, r_{0}=5 \Omega, \rho_{d}=0.1 \Omega$, and $\xi=60$. For $\Gamma \ll 1$, both $R_{D}^{x y}$ and $R_{D}^{x x}$ are small. As $\Gamma$ increases towards half, $R_{D}^{x x}$ increases dramatically while $R_{D}^{x y}$ remain almost unchanged.

for a given $\phi$ may be related to the coefficient at $\phi=0$ :

$$
R_{D}^{x x}[\Gamma, \phi]=R_{D}^{x x}[\Gamma, \phi=0] \frac{1+\eta \phi^{2}}{1+\phi^{2}},
$$

where $R_{D}^{x x}[\Gamma, \phi=0]$ is given in Eq. (8) and $\eta=\frac{L}{d} \frac{\Gamma}{1+\left(\frac{L}{d}\right) \Gamma}$. For $\Gamma \neq 0$ and $\frac{L}{d} \gg 1$, we find that $\eta \rightarrow 1$. Therefore, $R_{D}^{x x}[\Gamma, \phi] \rightarrow R_{D}^{x x}[\Gamma, \phi=0]$. We solved Eq. (B8) numerically for specific parameters and for different values of $\phi$. See Fig. 3(a). As evident, the longitudinal nonlocal drag resistivity is indifferent to the intralayer Hall effect.

For nonzero $\phi$, there is also a nonlocal transverse drag coefficient. The ratio between the longitudinal and the transverse drag coefficients is given by

$$
\frac{R_{D}^{x y}[\Gamma, \phi]}{R_{D}^{x x}[\Gamma, \phi]} \equiv \Phi=\frac{\phi}{1+\eta \phi^{2}} \frac{1-2 \Gamma}{1+\left(\frac{L}{d}\right) \Gamma} .
$$

For $\Gamma=0$ we find that $\Phi=\phi$, as expected. For $\Gamma \neq 0$ and $\frac{L}{d} \gg 1$ we find that $\Phi=\frac{\phi}{1+\phi^{2}} \frac{1-2 \Gamma}{1+\left(\frac{L}{d}\right) \Gamma}$. This ratio is plotted in Fig. 3(b).

Overall, there is a nonzero $R_{D}^{x y}$; however, it is parametrically smaller than $R_{D}^{x x}$. For $\Gamma \ll 1$, both $R_{D}^{x y}$ and $R_{D}^{x x}$ are small. As $\Gamma$ increases towards half, $R_{D}^{x x}$ increases dramatically while $R_{D}^{x y}$ remains almost unchanged.

\section{APPENDIX C: EVALUATION OF THE LOCAL DRAG RESISTANCE}

In order to evaluate the local drag resistivity, $\rho_{d}$, we treat the drag layer of the WSM (up to a distance of $d$ from one of surfaces) as an effective 2D layer with a known density $n_{d}=n_{3 \mathrm{D}} d$. We also assume that both graphene layers are doped away from the neutrality point, and have a finite density $n_{g}$.

Given two layers with densities $n_{g}$ and $n_{d}$ which are separated by a distance $\ell$, the drag resistivity may be obtained from the standard Kubo formula approach within the diagrammatic perturbation theory $[40,41]$ or from a memory function formalism [42]:

$$
\begin{aligned}
\rho_{d}= & \frac{\hbar}{16 e^{2} \pi n_{d} n_{g} T} \\
& \times \int \frac{d^{2} \mathbf{q} d \omega q^{2}}{(2 \pi)^{2}} \frac{\left|U_{12}(\mathbf{q}, \omega)\right|^{2} F_{1}(\mathbf{q}, \omega) F_{2}(\mathbf{q}, \omega)}{\sinh ^{2}\left(\frac{\hbar \omega}{2 T}\right)},
\end{aligned}
$$


where

$$
\begin{aligned}
F_{i}(\mathbf{q}, \omega) & =\operatorname{Im}\left[\Pi_{i}(\mathbf{q}, \omega)\right] \\
& =\int \frac{d^{2} \mathbf{k}}{(2 \pi)^{2}}\left(f_{F D}\left(\epsilon_{\mathbf{k}}\right)-f_{F D}\left(\epsilon_{\mathbf{k}+\mathbf{q}}\right)\right) \delta\left(\epsilon_{\mathbf{k}+\mathbf{q}}-\epsilon_{\mathbf{k}}-\hbar \omega\right)
\end{aligned}
$$

is the imaginary part of the is the single-layer retarded polarization operator and

$U_{12}^{-1}(\mathbf{q}, \omega)=e^{q \ell}\left(\frac{q}{2 \pi e^{2}}+\Pi_{1}+\Pi_{2}\right)+\Pi_{1} \Pi_{2} \frac{4 \pi e^{2}}{q} \sinh (q \ell)$

is the screened interlayer Coulomb interaction, where again $\Pi_{i}=\Pi_{i}(\mathbf{q}, \omega)$ is the single-layer retarded polarization operator.

For a two-dimensional, noninteracting electron gas in the ballistic regime the $F$ function is given by

$$
F_{i}(\mathbf{q}, \omega)=v_{i} \frac{\omega}{v_{f, i} q} \Theta\left(v_{f, i} q-\omega\right),
$$

where $v_{i}$ and $v_{f, i}$ are the density of states and the Fermi velocity in the $i$ th layer, respectively.

Additionally, in the limit of strong screening, the screened interlayer interaction can approximated by

$$
U_{12}(\mathbf{q}, \omega) \approx \frac{\pi e^{2}}{q_{T F, 1} q_{T F, 2}} \frac{q}{\sinh (q \ell)},
$$

where $q_{T F i}=2 \pi e^{2} v_{i}$ is the inverse Thomas-Fermi screening length.

Employing Eqs. (C4) and (C5), keeping in mind the degeneracies in each layer, the integrals in Eq. (C1) may be evaluated:

$$
\rho_{d}=\frac{h}{e^{2}} \frac{\zeta(3)}{256 \pi^{2}} \frac{T^{2}}{e^{4} \ell^{4}\left(n_{g} n_{d}\right)^{3 / 2}} .
$$

Denoting $\mathcal{N}=\left(n_{g} n_{d}\right)^{1 / 2}$, the local drag resistivity may be written as

$$
\rho_{d} \sim 4.43 \times 10^{6} \frac{T^{2}}{\mathcal{N}^{3} \ell^{4}}[\Omega],
$$

where $\mathcal{N}$ is measured in $\mathrm{cm}^{-2}, \ell$ in centimeters, and $T$ in kelvin. Finally, we may define the following typical dimensionless parameters (of the order of unity):

$$
\Delta=\frac{\mathcal{N}}{5 \times 10^{11} \mathrm{~cm}^{-2}}, \quad t=\frac{T}{100 \mathrm{~K}}, \quad \text { and } \quad \chi=\frac{\ell}{10 \mathrm{~nm}},
$$

which yields

$$
\rho_{d} \sim 0.35 \frac{t^{2}}{\Delta^{3} \chi^{4}}[\Omega]
$$

[1] A. M. Turner and A. Vishwanath, Beyond band insulators: Topology of semi-metals and interacting phases, arXiv:1301.0330.

[2] X. Wan, A. M. Turner, A. Vishwanath, and S. Y. Savrasov, Topological semimetal and Fermi-arc surface states in the electronic structure of pyrochlore iridates, Phys. Rev. B 83, 205101 (2011).

[3] A. A. Burkov and L. Balents, Weyl Semimetal in a Topological Insulator Multilayer, Phys. Rev. Lett. 107, 127205 (2011).

[4] P. Hosur and X. Qi, Recent developments in transport phenomena in Weyl semimetals, C. R. Phys. 14, 857 (2013).

[5] W. Witczak-Krempa and Y. B. Kim, Topological and magnetic phases of interacting electrons in the pyrochlore iridates, Phys. Rev. B 85, 045124 (2012).

[6] G. Xu, H. Weng, Z. Wang, X. Dai, and Z. Fang, Chern Semimetal and the Quantized Anomalous Hall Effect in $\mathrm{HgCr}_{2} \mathrm{Se}_{4}$, Phys. Rev. Lett. 107, 186806 (2011).

[7] H. Weng, C. Fang, Z. Fang, B. A. Bernevig, and X. Dai, Weyl Semimetal Phase in Noncentrosymmetric TransitionMetal Monophosphides, Phys. Rev. X 5, 011029 (2015).

[8] S.-M. Huang et al., A Weyl fermion semimetal with surface Fermi arcs in the transition metal monopnictide TaAs class, Nat. Commun. 6, 7373 (2015).

[9] M. Neupane et al., Observation of a three-dimensional topological Dirac semimetal phase in high-mobility $\mathrm{Cd}_{3} \mathrm{As}_{2}$, Nat. Commun. 5, 3786 (2014).

[10] Z. K. Liu et al., A stable three-dimensional topological Dirac semimetal $\mathrm{Cd}_{3} \mathrm{As}_{2}$, Nat. Mater. 13, 677 (2014).
[11] S.-Y. Xu, I. Belopolski, N. Alidoust, M. Neupane, G. Bian, C. Zhang, R. Sankar, G. Chang, Z. Yuan, C.-C. Lee, S.-M. Huang, H. Zheng, J. Ma, D. S. Sanchez, B. Wang, A. Bansil, F. Chou, P. P. Shibayev, H. Lin, S. Jia, and M. Z. Hasan, Discovery of a Weyl fermion semimetal and topological Fermi arcs, Science 349, 613 (2015).

[12] B. Q. Lv, H. M. Weng, B. B. Fu, X. P. Wang, H. Miao, J. Ma, P. Richard, X. C. Huang, L. X. Zhao, G. F. Chen, Z. Fang, X. Dai, T. Qian, and H. Ding, Experimental Discovery of Weyl Semimetal TaAs, Phys. Rev. X 5, 031013 (2015).

[13] Z. K. Liu et al., Discovery of a three-dimensional topological Dirac semimetal, $\mathrm{Na}_{3} \mathrm{Bi}$, Science 343, 864 (2014).

[14] S. Jeon et al., Landau quantization and quasiparticle interference in the three-dimensional Dirac semimetal $\mathrm{Cd}_{3} \mathrm{As}_{2}$, Nat. Mater. 13, 851 (2014).

[15] H. Yi et al., Evidence of topological surface state in threedimensional Dirac semimetal $\mathrm{Cd}_{3} \mathrm{As}_{2}$, Sci. Rep. 4, 6106 (2014).

[16] B. Q. Lv, N. Xu, H. M. Weng, J. Z. Ma, P. Richard, X. C. Huang, L. X. Zhao, G. F. Chen, C. E. Matt, F. Bisti, V. N. Strocov, J. Mesot, Z. Fang, X. Dai, T. Qian, M. Shi, and H. Ding, Observation of Weyl nodes in TaAs, Nat. Phys. 11, 724 (2015).

[17] C.-L. Zhang, Z. Yuan, Q.-D. Jiang, B. Tong, C. Zhang, X. C. Xie, and S. Jia, Tantalum monoarsenide: An exotic compensated semimetal, Phys. Rev. B 95, 085202 (2017).

[18] R. Batabyal, N. Morali, N. Avraham, Y. Sun, M. Schmidt, C. Felser, A. Stern, B. Yan, and H. Beidenkopf, Visualizing weakly 
bound surface Fermi arcs and their correspondence to bulk Weyl fermions, Sci. Adv. 2, e1600709 (2016).

[19] H. Inoue, A. Gyenis, Z. Wang, J. Li, S. W. Oh, S. Jiang, N. Ni, B. A. Bernevig, and A. Yazdani, Quasiparticle interference of the Fermi arcs and surface-bulk connectivity of a Weyl semimetal, Science 351, 1184 (2016).

[20] D. T. Son and B. Z. Spivak, Chiral anomaly and classical negative magnetoresistance of Weyl metals, Phys. Rev. B 88, 104412 (2013).

[21] X. Huang, L. Zhao, Y. Long, P. Wang, D. Chen, Z. Yang, H. Liang, M. Xue, H. Weng, Z. Fang, X. Dai, and G. Chen, Observation of the Chiral-Anomaly-Induced Negative Magnetoresistance in 3D Weyl Semimetal TaAs, Phys. Rev. X 5, 031023 (2015).

[22] C.-L. Zhang et al., Signatures of the Adler-Bell-Jackiw chiral anomaly in a Weyl fermion semimetal, Nat. Commun. 7, 10735 (2016).

[23] A. C. Potter, I. Kimchi, and A. Vishwanath, Quantum oscillations from surface fermi arcs in weyl and dirac semimetals, Nat. Commun. 5, 5161 (2014).

[24] P. J. W. Moll, N. L. Nair, T. Helm, A. C. Potter, I. Kimchi, A. Vishwanath, and J. G. Analytis, Transport evidence for Fermiarc-mediated chirality transfer in the Dirac semimetal $\mathrm{Cd}_{3} \mathrm{As}_{2}$, Nature (London) 535, 266 (2016).

[25] S. A. Parameswaran, T. Grover, D. A. Abanin, D. A. Pesin, and A. Vishwanath, Probing the Chiral Anomaly with Nonlocal Transport in Three-Dimensional Topological Semimetals, Phys. Rev. X 4, 031035 (2014).

[26] Y. Baum, E. Berg, S. A. Parameswaran, and A. Stern, Current at a Distance and Resonant Transparency in Weyl Semimetals, Phys. Rev. X 5, 041046 (2015).

[27] B. N. Narozhny and A. Levchenko, Coulomb drag, Rev. Mod. Phys. 88, 025003 (2016).

[28] A. G. Rojo, Electron-drag effects in coupled electron systems, J. Phys.: Condens. Matter 11, R31 (1999).

[29] P. M. Solomon, P. J. Price, D. J. Frank, and D. C. La Tulipe, New Phenomena in Coupled Transport Between 2D and 3D Electron-Gas Layers, Phys. Rev. Lett. 63, 2508 (1989).

[30] T. J. Gramila, J. P. Eisenstein, A. H. MacDonald, L. N. Pfeiffer, and K. W. West, Mutual Friction Between Parallel
Two-Dimensional Electron Systems, Phys. Rev. Lett. 66, 1216 (1991).

[31] U. Sivan, P. M. Solomon, and H. Shtrikman, Coupled ElectronHole Transport, Phys. Rev. Lett. 68, 1196 (1992).

[32] R. V. Gorbachev, A. K. Geim, M. I. Katsnelson, K. S. Novoselov, T. Tudorovskiy, I. V. Grigorieva, A. H. MacDonald, S. V. Morozov, K. Watanabe, T. Taniguchi, and L. A. Ponomarenko, Strong Coulomb drag and broken symmetry in double-layer graphene, Nat. Phys. 8, 896 (2012).

[33] W.-K. Tse, B. Y.-K. Hu, and S. D. Sarma, Theory of Coulomb drag in graphene, Phys. Rev. B 76, 081401 (2007).

[34] M. Gibertini, A. Tomadin, F. Guinea, M. I. Katsnelson, and M. Polini, Electron-hole puddles in the absence of charged impurities, Phys. Rev. B 85, 201405 (2012).

[35] M. Carrega, T. Tudorovskiy, A. Principi, M. I. Katsnelson, and M. Polini, Theory of Coulomb drag for massless Dirac fermions, New J. Phys. 14, 063033 (2012).

[36] J. C. W. Song, D. A. Abanin, and L. S. Levitov, Coulomb drag mechanisms in graphene, Nano Lett. 13, 3631 (2013).

[37] T. Liang, Q. Gibson, M. N. Ali, M. Liu, R. J. Cava, and N. P. Ong, Ultrahigh mobility and giant magnetoresistance in the dirac semimetal $\mathrm{Cd}_{3} \mathrm{As}_{2}$, Nat. Mater. 14, 280 (2015).

[38] C. Shekhar et al., Extremely large magnetoresistance and ultrahigh mobility in the topological Weyl semimetal candidate NbP, Nat. Phys. 11, 645 (2015).

[39] Z. Wang, Y. Zheng, Z. Shen, Y. Lu, H. Fang, F. Sheng, Y. Zhou, X. Yang, Y. Li, C. Feng, and Z.-A. Xu, Helicity-protected ultrahigh mobility Weyl fermions in NbP, Phys. Rev. B 93, 121112 (2016).

[40] A. Kamenev and Y. Oreg, Coulomb drag in normal metals and superconductors: Diagrammatic approach, Phys. Rev. B 52, 7516 (1995).

[41] K. Flensberg and B. Y.-K. Hu, Plasmon enhancement of Coulomb drag in double-quantum-well systems, Phys. Rev. B 52, 14796 (1995).

[42] L. Zheng and A. H. MacDonald, Coulomb drag between disordered two-dimensional electron-gas layers, Phys. Rev. B 48, 8203 (1993). 